\title{
The shift from a response strategy to object-in-place strategy during learning is accompanied by a matching shift in neural firing correlates in the hippocampus
}

\author{
Inah Lee ${ }^{1}$ and Jangjin Kim \\ Department of Brain and Cognitive Sciences, Seoul National University, Seoul 151-746, Korea
}

\begin{abstract}
Hippocampal-dependent tasks often involve specific associations among stimuli (including egocentric information), and such tasks are therefore prone to interference from irrelevant task strategies before a correct strategy is found. Using an object-place paired-associate task, we investigated changes in neural firing patterns in the hippocampus in association with a shift in strategy during learning. We used an object-place paired-associate task in which a pair of objects was presented in two different arms of a radial maze. Each object was associated with reward only in one of the arms, thus requiring the rats to consider both object identity and its location in the maze. Hippocampal neurons recorded in CAl displayed a dynamic transition in their firing patterns during the acquisition of the task across days, and this corresponded to a shift in strategy manifested in behavioral data. Specifically, before the rats learned the task, they chose an object that maintained a particular egocentric relationship with their body (response strategy) irrespective of the object identity. However, as the animal acquired the task, it chose an object according to both its identity and the associated location in the maze (object-in-place strategy). We report that CAl neurons in the hippocampus changed their prospective firing correlates according to the dominant strategy (i.e., response versus object-in-place strategy) employed at a given stage of learning. The results suggest that neural firing pattern in the hippocampus is heavily influenced by the task demand hypothesized by the animal and the firing pattern changes flexibly as the perceived task demand changes.
\end{abstract}

[Supplemental material is available online at http://www.learnmem.org.]

An event often involves objects placed in particular locations. To form a cognitive representation of such an event, an animal needs to represent many heterogeneous pieces of information such as individual objects, their spatial locations, and also egocentric body movements associated with the navigation through the objects and locations. All the information then needs to be associated in a correct way (e.g., proper object-place association) for successful representation of the event. Processing only a subset of information successfully may lead to an error in identifying the correct event. It is believed that the hippocampus is essential for forming and retrieving such conjunctive representations among stimuli for processing events (O'Reilly and McClelland 1994).

Due to its conjunctive information processing after receiving inputs from multiple sources (e.g., information for object recognition, egocentric memory, allocentric memory, etc.), the hippocampal system is prone to competition with other systems especially when a simpler strategy that requires information from a single source seems a viable option. Behavioral and pharmacological studies have supported this hypothesis (Packard and McGaugh 1996; Poldrack and Packard 2003). Physiologically, the influence of switching strategy on hippocampal neuronal firing has been documented in the literature and often tested in a plus maze. It has been shown, for example, that passing a common area of space using different task demands alters the firing properties of hippocampal neurons (Wood et al. 2000; Ferbinteanu and Shapiro 2003; Lee et al. 2006; Eschenko and Mizumori 2007). In most of these studies, however, rats were pre-trained before

${ }^{1}$ Corresponding author.

E-mail inahlee@snu.ac.kr; fax 82-2-883-8014.

Article is online at http://www.learnmem.org/cgi/doi/10.1101//m.1829110. electrophysiological recordings were made and an experimenter enforced the change in task demand. Therefore, it has been difficult to investigate a naturally occurring competition between different strategies as learning proceeds and the resulting neural dynamics in the hippocampus especially when learning takes place for the first time.

Using a hippocampal dependent task, we aimed to characterize such naturally occurring changes in hippocampal firing patterns as a wrong strategy is replaced by the correct strategy during learning. We also investigated the relationships between the changes in firing patterns and behavioral performance. We used a biconditional object-place paired-associate task for this purpose. We demonstrated previously that hippocampal lesioned rats were severely impaired in this task (Lee and Solivan 2008). In this task, two different objects are presented simultaneously as a pair, and rats are required to discriminate between the objects according to the location (an arm in a radial maze) in which they are presented. Once it enters an arm, the rat positions itself between two objects and must choose either the left or right direction toward either object to displace the object for obtaining a food reward. Since the relative object positions within an arm vary across trials, remembering the running direction in which a reward is found (i.e., response strategy) is meaningless and the rat must learn to pay attention to the object identity and its associated location in space (i.e., object-in-place strategy). In our previous studies (Lee and Solivan 2008, 2010; Jo and Lee 2010), we observed a strong competition in this task between a response strategy and object-in-place strategy. Normal rats used the response strategy before learning occurred, but exhibited a sharp transition to the object-in-place strategy in several days. The selective involvement of the hippocampus was strongly implicated in 
the task as the hippocampal lesioned rats show stereotypic egocentric responses (Lee and Solivan 2008). The current study aimed to find and characterize in detail matching neural correlates in the hippocampus in the object-place paired-associate task.

\section{Results}

\section{Learning curves in an object-place paired-associate task}

Three rats implanted with multi-tetrode recording devices were trained in a biconditional object-place paired-associate task (Fig. 1A), in which two objects (toy girl and cylinder denoted as $\mathrm{G}$ and $\mathrm{C}$, respectively, in Fig. 1A) were simultaneously presented on the left and right sides (counterbalanced) in a so-called choice platform (Lee and Solivan 2008). One of the objects was associated with a reward as they were simultaneously presented in one of two places (either arm 3 or arm 5) in a radial maze positioned at the center of a curtained environment with distinct visual cues. In other words, the toy girl (G) was always rewarded in arm 3, but not in arm 5, and the cylinder (C) was rewarded in arm 5, but not in arm 3. The location associated with the rewarding object
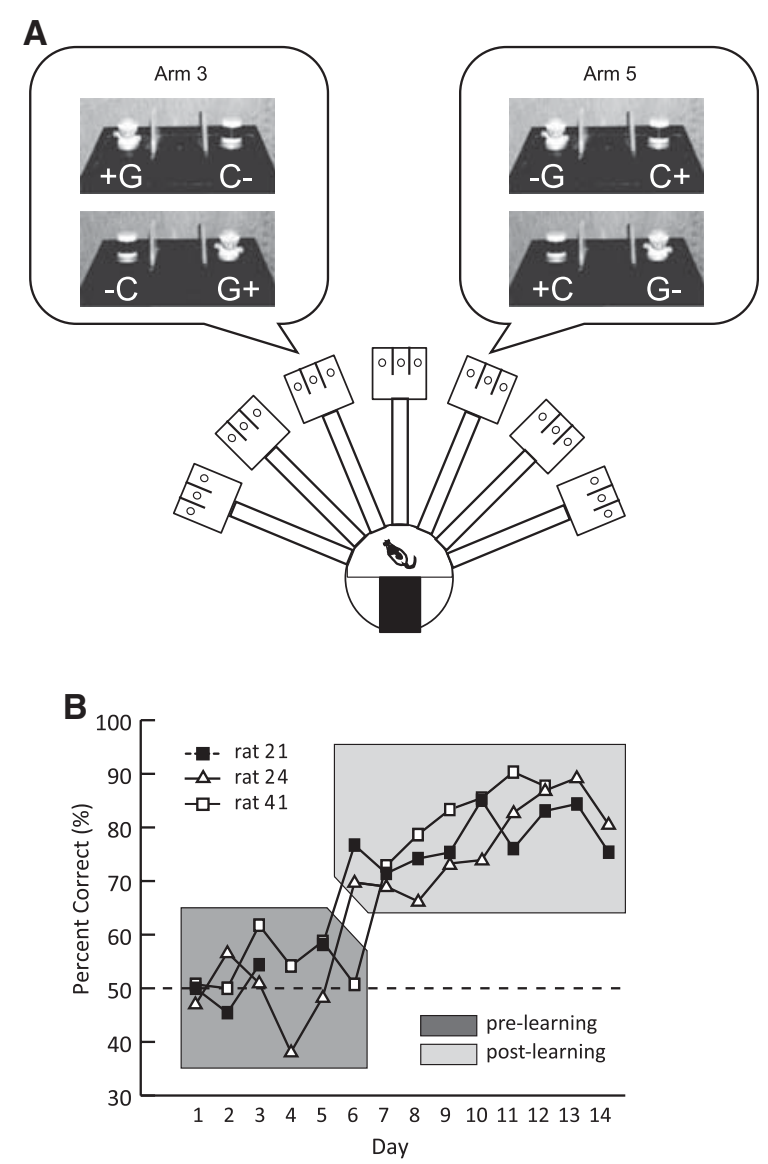

Figure 1. Acquisition of the object-place paired-associate task. (A) Overview of the radial arm maze. The start box is shown as a black rectangle. Insets illustrate possible spatial configurations of the objects (G: toy girl, C: cylinder) and correct choices (+: reward, -: no reward) in the choice platform in either arm 3 or arm 5. (B) Learning curves of all animals across days. Chance-level performance (50\%) is denoted by the dotted line. The acquisition periods before and after the rats exceeded the criterion are indicated by a dark gray area and light gray area, respectively. Data for Day 4 in rat 21 and Days 13 and 14 in rat 41 are unavailable due to technical problems during data collection. in a given platform was counterbalanced between left and right food wells and displacing a correct object revealed a quarter piece of cereal reward in the food well.

The animals exhibited remarkably similar learning curves in the current task with little variability among them throughout the two-week training period (Fig. 1B). Compared to their initial chance-level performance in the earlier learning period (prelearning period), all three animals successfully performed the task to criterion of $70 \%$ or above after 5-6 days of training (postlearning period). The performance levels were significantly different between the pre- and post-learning periods $\left(t_{(39)}=11.9, P<\right.$ $0.0001)$. Naïve rats initially tended to choose the food well on either the left or right side once entering the choice platform regardless of the associated object during the pre-learning period (Supplemental Fig. 1), which resulted in chance-level performance. However, their performance improved sharply (Fig. 1B) as the rats inhibited such rule-irrelevant, response strategy across training days (Supplemental Fig. 1) and gradually increased to an asymptotic level of $80 \%-90 \%$ as they used the object-in-place strategy.

\section{Overall firing characteristics of hippocampal neurons in object-place paired association}

Multiple CA1 neurons in the hippocampus were recorded simultaneously from eight tetrodes (Supplemental Fig. 2) in each animal throughout the entire learning period of 12-14 days, and putative CA1 complex-spike units were isolated offline. Among those, only neurons $(n=824)$ that fired in the arms of the maze were included for analysis and only outbound journeys (from entering the arm to displacement of an object) were considered ( $n=21.1$ cells recorded per day per animal on average, ranging from $n=4$ to 50; median $n=19$; Table 1 ). Most CA1 neurons fired in a spatially localized fashion in the maze (Fig. 2A). The proportional distributions of the firing-field locations of CA1 neurons were quantified according to their center-of-mass locations in the maze after each arm was arbitrarily divided into discrete zones (Fig. 2B). The largest proportion of firing fields (65\%) was found closer to the choice platform area where critical events (i.e., choosing an object) occurred (45\% in the distal arm region and 20\% in the junction between the distal arm and choice platform). The relative proportions of neurons representing different zones were not significantly different between pre- and post-learning periods except for the proportion of neurons firing in the proximal and distal arm regions. When compared between the pre- and post-learning periods, the proportion of neurons representing the proximal arm region increased (vice versa for the distal arm region) as rats learned the task (Fig. 2B). When a $\chi^{2}$ test was used to compare the relative proportions of the distal arm- and proximal armrepresenting neurons between pre- and post-learning periods, there was a trend of proportional differences between the two periods $\left(\chi^{2}=3.71, P=0.05\right)$.

The current task required the animals to process both object and place information concurrently and we examined if hippocampal neurons represented either object or place information in a relatively independent manner as suggested in primate studies (Eifuku et al. 1995; Rolls et al. 2005). A selectivity index (0-1 for minimum to maximum score) for either a specific place (armselectivity index) or an object (object-selectivity index) was calculated (Fig. 2C). Approximately $80 \%$ of neurons showed an index of 0.6 or higher when the arm-selectivity index was calculated between arms 3 and 5, demonstrating that neuronal firing in CA1 was significantly modulated by global spatial information (O'Keefe and Dostrovsky 1971; Muller et al. 1987). In contrast, neuronal activity in CA1 was not particularly tied to the object variable per se (independent of the space or response variable) 
Table 1. The numbers of neurons used for analyses for individual rats across days

\begin{tabular}{|c|c|c|c|c|c|c|c|c|c|c|c|c|c|c|}
\hline & \multicolumn{14}{|c|}{ Day } \\
\hline & 1 & 2 & 3 & 4 & 5 & 6 & 7 & 8 & 9 & 10 & 11 & 12 & 13 & 14 \\
\hline Rat 21 & 38 & 20 & 24 & $\mathrm{n} / \mathrm{a}$ & 14 & 11 & 23 & 24 & 16 & 13 & 20 & 38 & 24 & 19 \\
\hline Rat 24 & 16 & 6 & 4 & 5 & 7 & 11 & 4 & 17 & 15 & 13 & 17 & 7 & 17 & 12 \\
\hline Rat 41 & 36 & 35 & 40 & 19 & 28 & 42 & 50 & 44 & 22 & 30 & 24 & 19 & $n / a$ & $\mathrm{n} / \mathrm{a}$ \\
\hline Average & 30.0 & 20.3 & 22.7 & 12.0 & 16.3 & 21.3 & 25.7 & 28.3 & 17.7 & 18.7 & 20.3 & 21.3 & 20.5 & 15.5 \\
\hline
\end{tabular}

as measured by the object-selectivity index. Furthermore, object configuration in the choice platform alone could not explain the specific firing patterns of CA1 neurons in the current task. The result remained the same when only the firing fields in the choice platforms were considered (data not shown). Since the rats showed a response bias toward a food well on a particular side (either left or right) during the pre-learning period, we also measured the response-selectivity index to gauge the degree to which CA1 neurons fire in association with a certain turning direction independent of object identity and arm information. CA1 neuronal firing did not fire exclusively just based on response bias in the current task (Fig. 2C). The selectivity patterns for place, object, object configuration, and response variables remained largely unchanged between pre- and post-learning periods (all $P$-values $>0.05$, Kolmogorov-Smirnov test; Supplemental Fig. 3). These results suggest that the spatial variable (i.e., arm) among
A

A Cell 1

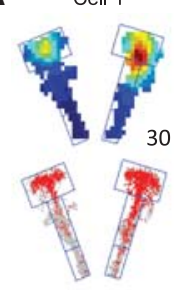

Cell 8

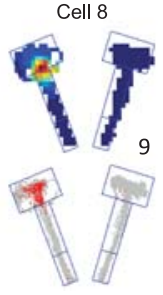

Cell 2

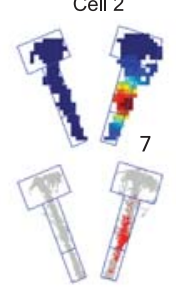

Cell 9

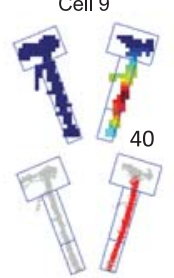

B
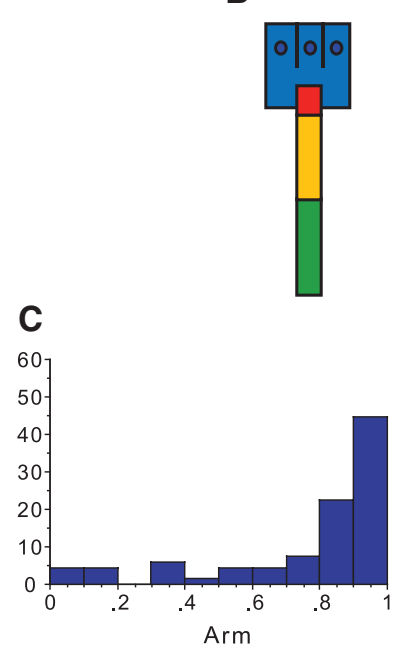

Cell 3

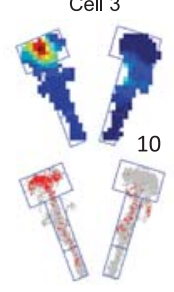

Cell 10

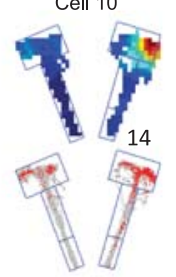

Overall
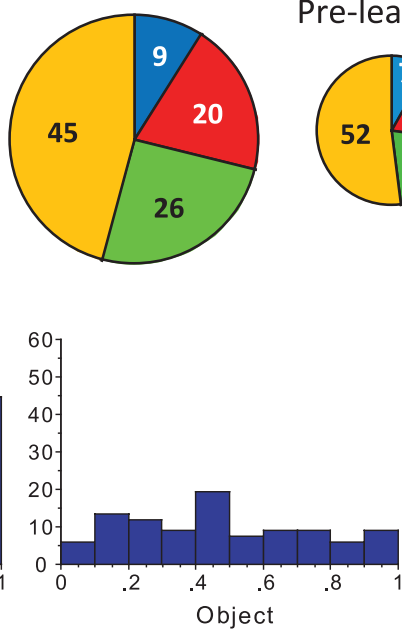

Cell 4

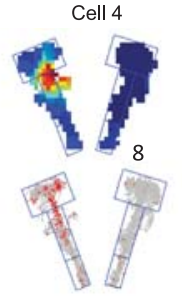

Cell 11

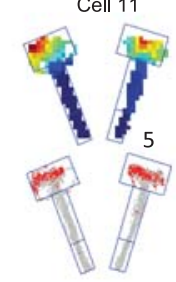

Cell 5
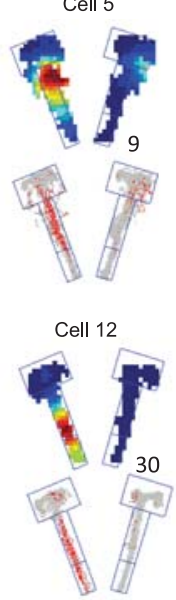

Cell 6
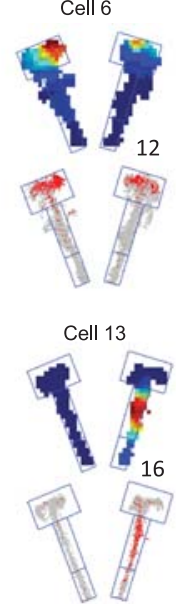

Cell 7
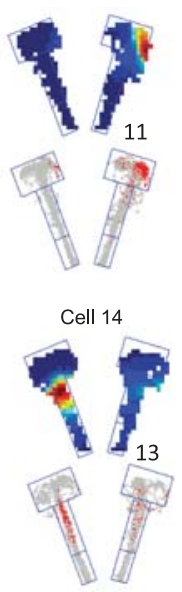

Pre-learning Post-learning
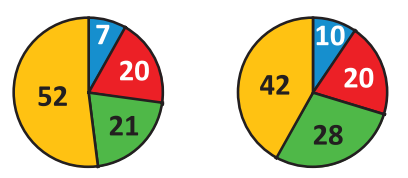

achoice platform

adistal arm-choice platform junction

口 proximal arm

udistal arm
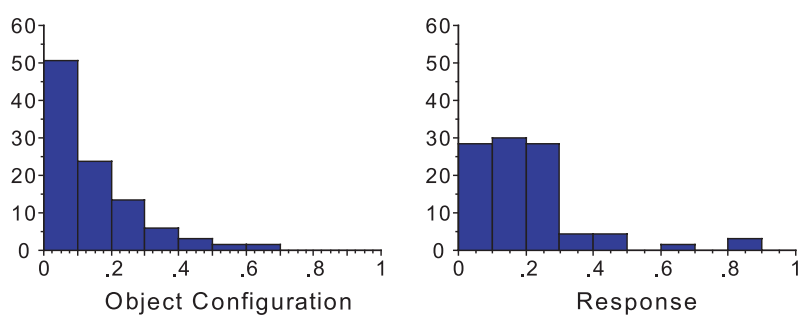

Figure 2. Firing characteristics of hippocampal neurons in object-place paired association. (A) Representative examples of average spatial firing patterns of CA1 neurons in the maze (center platform excluded). Cells shown were not recorded simultaneously. (Upper) Color-coded firing rate map (maximum firing rate in hertz shown as number). (Lower) Raw spiking positions (red) overlaid on position traces (gray). Physical boundaries of arms 3 and 5 are shown in each example. (B) The maze arm was divided into arbitrary zones (color-coded). The proportion of CA1 neurons whose firing field locations (COM) were found in different zones are shown as pie charts for the entire training period (Overall) as well as separately for both pre- and post-learning periods. Numbers show percent. (C) Proportional distributions of CA1 neurons with respect to their firing correlates with a specific arm, object, object configuration, or bias toward a specific turning direction. 
others was the strongest factor that determined the global firing patterns of hippocampal neurons in the maze environment.

\section{Emergence of object-in-place strategy and its associated firing patterns during learning}

To find dynamic neural changes correlated with a shift in strategy during learning, individual trials associated with each arm were categorized into discrete "trial conditions" considering (1) the relative locations of the objects in the choice platform (i.e., object configuration) and (2) the direction of the response made toward one of the objects (i.e., response bias). As shown in Figure 3A, all trials were categorized based on the combination of these two factors. Correct responses were made when the animal's turning direction matched the rewarding object's location (which inevitably required the opposite turning direction for the other object configuration in the same arm) as shown in Figure 3A (the green pair of trial conditions showing object-in-place trials). Incorrect choices were made when the rat turned toward only one side of the choice platform without considering the object associated with the directed location (the red pairs indicating response trial conditions).

The inverse relationship between the response strategy and object-in-place strategy across learning was obvious when a bias index was calculated for each strategy (Fig. 3B). The bias index for response was calculated by taking the absolute value after subtracting the number of choices made for the right food well from the number of choices made for the left food well and then dividing the result by the total number of choices. A bias index for the correct object was also calculated similarly by subtracting the number of choices made for the incorrect object in the arm from the number of choices made for the correct object, and dividing by the total number of choices. The line graphs in Figure 3B were obtained by averaging each index calculated for arm 3 and arm 5. As learning proceeded, the bias toward a certain direction diminished, whereas the preference for the target object associated with a given arm dramatically increased over time. A repeated-measures ANOVA revealed a highly significant interaction between the two bias indices $\left(F_{(10,20)}=7.0, P<0.0001\right)$.

As shown in Figure 3C, the neural firing patterns were correlated with the behavioral crossing patterns between the two strategies shown in Figure 3B. Specifically, important differences were
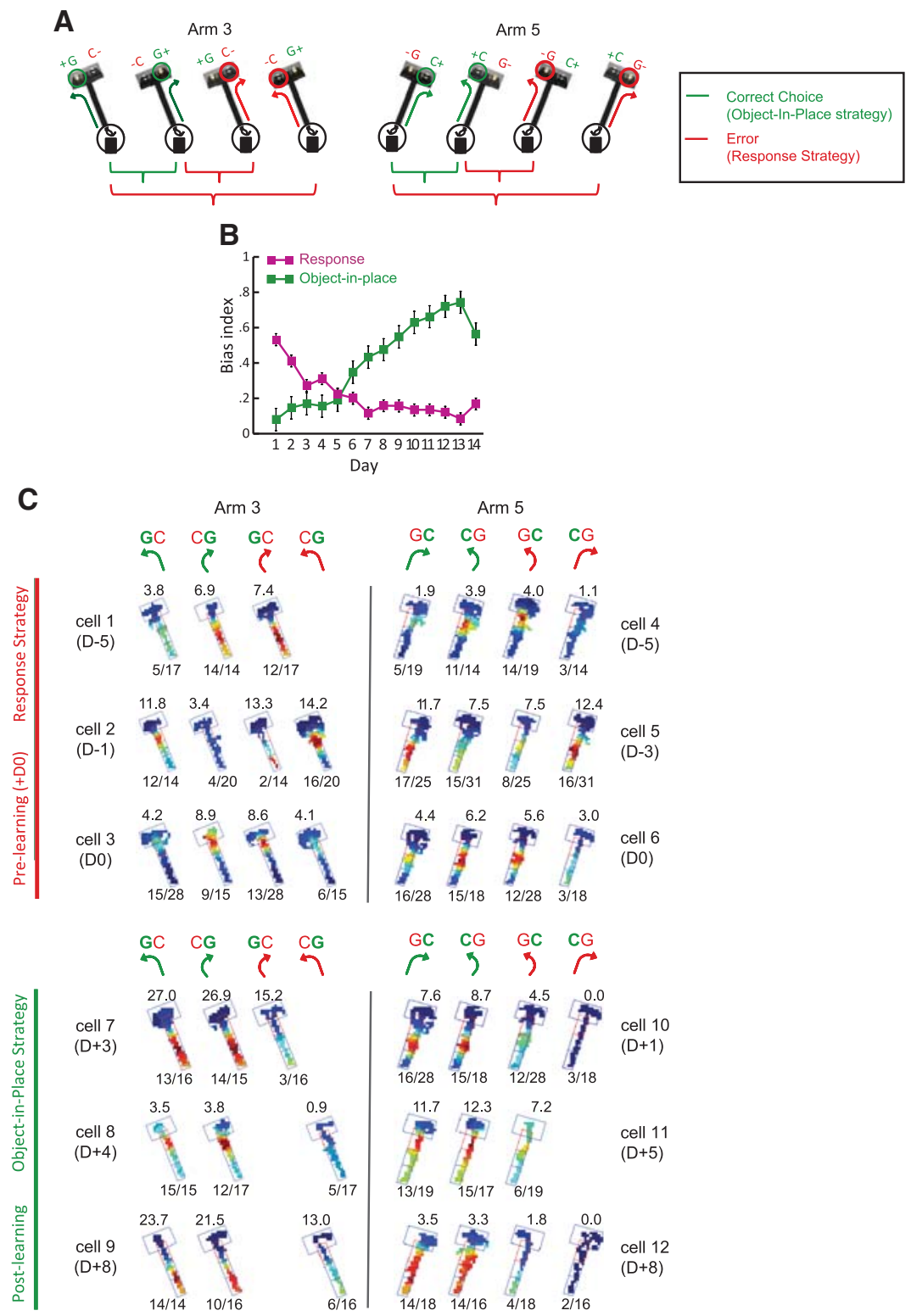

Figure 3. Differential firing patterns before and after the object-place paired-associate learning. $(A)$ Categorical parsing of trials for analytical purposes considering the relative locations of the objects in the choice platform and turning directions (shown as arrows). Green and red circles over objects indicate objects displaced correctly and incorrectly, respectively. Correct choice (object-in-place strategy): A pair of trial conditions in which the rat made rule-relevant choices (resulting in opposite turning directions between different object configurations); Error (response strategy): A pair of trial conditions in which the animals made rule-irrelevant choices (showing the same turning direction). (B) The relationship between the object-in-place bias (the preference for the target object regardless of the associated turning directions) and the response bias as learning proceeded across days (error bar $=$ mean \pm S.E.M.). (C) Representative examples of firing rate maps of neurons recorded in either arm 3 or arm 5 during the pre-learning period (cells 1 to 6, upper panel) and the ones recorded in the post-learning period (cells 7 to 12 , lower panel). Cells shown were not necessarily recorded simultaneously. D0 indicates the first day when the rats reached the criterion performance level. For each neuron, the overall firing pattern was broken down into individual rate maps for different trial conditions following the scheme shown in $A$. The number over each rate map shows the maximum firing rate (hertz) and the number below the rate map indicates the number of trials for a given trial condition for the total number of trials performed with the specific object-arm paired configuration. Only the trial conditions with more than two trials/conditions are shown.

found between pre- and post-learning periods when spatial firing rate maps for individual neurons were constructed separately for different trial conditions following the trial-categorizing scheme shown in Figure 3A. As shown in Figure 3C, in the 
pre-learning period, the prospective firing patterns of CA1 neurons before the rats reached the choice platform were more similar to each other between trial conditions that shared the same turning direction (e.g., between CG-right turn and GC-right turn conditions in cell 3; the two rate maps in the middle) than ones that required the object-in-place strategy (e.g., between GC-left turn and CG-right turn conditions in cell 3; see the first two rate maps from the left). In contrast, in the post-learning period, CA1 neurons showed similar firing patterns between objectin-place trial conditions (e.g., between GC-right turn and CGleft turn conditions in cell 10; see the first two rate maps from the left) in which the rat chose a correct object regardless of its associated turning directions within a given arm.

To quantitatively describe the relationship between neuronal firing and learning strategy, the degree of similarity between the spatial firing patterns for different task conditions (Fig. 4A) was measured by calculating the Pearson's correlation coefficient between firing rate maps either for the object-in-place trial conditions (Fig. 4A, right) or for the trials in which the rat turned to the object on the same side with respect to its body (both left- and right-turn pairs were analyzed and the bigger correlation coefficient of the two was taken as a conservative measure) as illustrated in the left panel in Figure 4A (only arm 3 and left-turn bias examples are shown for illustrative purposes). The following constraints were imposed to control possible confounding factors that may be associated with the correlation analysis. First, each maze arm was narrowly designed $(8 \mathrm{~cm})$ to maximize trajectory overlap and the cross-correlation analyses throughout the study were restricted to the areas with overlapping trajectories (resulting in the exclusion of the choice platforms and center platform). To verify the overlap in position traces (Supplemental Fig. 4A), the position coordinates for different conditions (GC and CG conditions) were rotated so that position traces for both arms were aligned vertically (counterclockwise for arm 5 and clockwise for arm 3; Supplemental Fig. 4B). The distributions of position trace were then compared between the object-in-place conditions (GC and CG) using $t$-test. All statistical tests were not significant $(P$-values $>0.05)$ except for one day (rat $41^{\prime}$ s d 8 data for the GC-CG trial condition in arm $\left.5, t_{(18)}=2.43, P<0.05\right)$. Even for that day, the distribution range for the position trace was not significantly different across the task conditions, but the significant difference stemmed from the fact that the animal stayed in the middle of the arm significantly longer than usual in the first trial. Once that trial was removed, the position distributions between object-in-place conditions were the same. Second, when constructing firing rate maps, an adaptive binning algorithm was
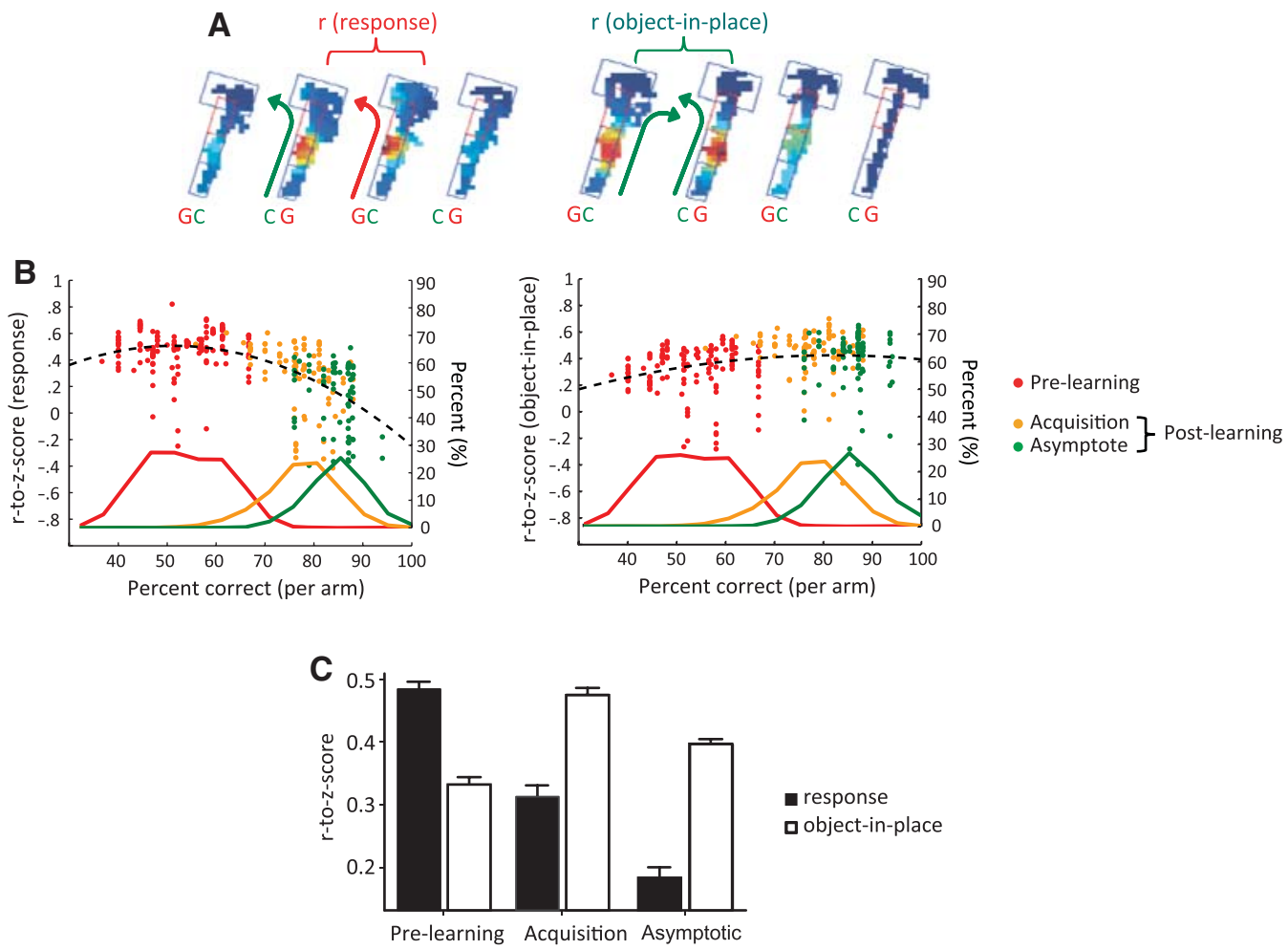

Figure 4. Increased similarity in CA1 neuronal firing patterns between rule-relevant trial conditions parallel the increase in performance in the objectplace paired-associate task. (A) The similarity of spatial firing patterns of a neuron was measured between different trial conditions by calculating the pixel-by-pixel Pearson's correlation coefficient between the rate maps separately constructed from those conditions. Examples showing how to calculate the correlations between the rate maps for response strategy-based trials (left) and object-in-place strategy-based trials (right) are shown (arm 5). Both leftand right-turn paired trials were analyzed and the bigger one was taken for the regression analysis (only left-turn pairs were shown in the figure for illustrative purpose). Arrows indicate turning directions of the animals in those trials types. Green and red colors for arrows and object abbreviations indicate correct and incorrect choices, respectively. (B) Bivariate scatter plots showing the relationship between performance and the similarity in firing patterns between response trials (left) or object-in-place trials (right). To calculate the similarity in firing pattern, cross-correlation was performed between the firing rate maps. Correlation coefficient was then normalized using Fisher's r-to-z transformation. The dotted lines indicate polynomial fitting lines with order 2. Scatter points were color-coded on the basis of learning stages and color-matched, proportional distributions of behavioral performance data were shown along the $x$-axis to illustrate the time course of the firing correlates across learning. The second $y$-axis on the right side of the figure is to indicate the proportional distribution of behavioral performance. (C) Fisher's r-to-z transformation of the correlation coefficients calculated between rate maps. As shown in $B$, the similarity in firing patterns between object-in-place trials increased in the post-learning period (acquisition and asymptote) and the reverse trend was observed in response-based trials. 
used to balance between sampling error and spatial resolution (Jung et al. 1994; Skaggs et al. 1996; Lee et al. 2004a,b). The adaptive binning algorithm was proven to be effective in solving tradeoffs between sampling error and blurring error stemming from representing a relatively large physical space by pixels. Visual cross-examinations of raw spiking patterns on the maze in comparison to their associated rate maps also confirmed this in our study. Finally, only rate maps constructed based on more than two trials (per condition) were considered in the correlation analysis and we verified the results by running the same analysis using a more stringent criterion (i.e., minimum number of trials per condition $\geq 5$ ).

A nonparametric correlation analysis using Spearman rank correlation (Fig. 4B) showed that animal's performance level reliably predicted the amount of similarity between the firing patterns in different trial conditions. For example, transformed correlation coefficients (using Fisher's r-to-z) were high between response trial conditions ( $\left.r_{\text {response }}\right)$ and low between objectin-place trial conditions ( $\left.r_{\text {object-in-place }}\right)$ when performance was lower, whereas this trend was reversed as performance increased $\left(r_{\text {response }}=-0.62, \quad P_{\text {response }}<0.001\right.$ and $r_{\text {object-in-place }}=0.36$, $\left.P_{\text {object-in-place }}<0.001\right)$. As color-coded in Figure $4 \mathrm{~B}$, data points (red) showing a high level of correlation in $r_{\text {response }}$ values were associated with the pre-learning period and these points showed

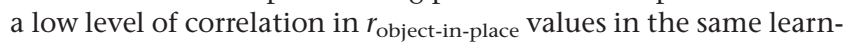
ing period. Data points (orange and green) showing opposite patterns in the post-learning period demonstrated a transition between strategies across pre- and post-learning periods. Significant correlations were also found when the minimal requirement for the number of sampling of trials was raised to five trials from two trials (all $P$-values $<0.001$ ). To examine learning stage-related dynamics in more detail, we divided the postlearning period into the initial acquisition stage ("acquisition" in Fig. 4B) and the asymptotic performance stage ("asymptote" in Fig. 4B) with the criterion of $80 \%$ performance level. This criterion categorized the data points from the post-learning period reasonably well. (In rat 21, days 6-11 and day 14 belonged to the acquisition stage and days 12 and 13 were categorized into the asymptotic performance stage. In rat 24 , days $6-10$ belonged to the acquisition and the rest in the asymptote. In rat 41 , days 7 and 8 belonged to the acquisition and the rest of the days in the asymptote.) The percentage shown on the second $y$-axis is for the distribution of performance range (shown as colored line graphs) associated with scatter points. This is to show both performance level and correlational relationship between the spatial firing patterns and strategy simultaneously in a single graph. Thus, Figure $4 \mathrm{~B}$ shows that it was more likely to observe the neurons that fired more dissimilarly between response-strategy conditions (but more similarly between object-in-place conditions) as performance improved. As shown in Figure 4B, it appears that the correlation between spatial firing rate maps representing response trial conditions dropped significantly as the performance improved, whereas the correlation between spatial representations for object-in-place conditions exhibited a rather linear increase with the improvement in performance. This suggests that the reduction of hippocampal firing correlates with the response bias should serve as a good predictor for the acquisition of the task.

The main results were also confirmed by an ANOVA performed on rate-map similarity after Fisher's r-to-z transformation (Fig. 4C), showing a highly significant interaction between the learning stage (the pre-learning stage $[n=104]$ and the postlearning stage divided into acquisition stage $[n=50]$ and asymptote stage $[n=32])$ and the strategies associated with different trial conditions $\left(F_{(2,183)}=61.9, P<0.01\right)$. Post-hoc paired comparisons between Z-scores for different learning stages revealed highly significant differences as follows: All pairwise comparisons between Z-scores from the object-in-place and response conditions were highly significant $(P$-values $<0.01)$. Within the response strategy, $P$-values were of $<0.01$ between pre-learning and acquisition and between acquisition and asymptote. The $P$-value was $<0.05$ between pre-learning and asymptote. Within object-in-place strategy, $P$-value was $<0.01$ between pre-learning and acquisition, and $P$-values were $<0.05$ between acquisition and asymptote as well as between pre-learning and asymptote. These results showed that neuronal firing patterns between trial conditions associated with response strategy became less similar as rats acquired the task, whereas the opposite trend was true for object-in-place strategy. With respect to the significant decrease in similarity in firing patterns between acquisition and asymptote stages for the object-in-place strategy, seven outliers (outside 3 standard deviations from the mean) in the asymptotic stage produced the significant difference. Without those outliers (thus with data points within 3 standard deviations), there was no significant difference between acquisition and asymptote stages for object-in-place strategy.

Since the number of trials associated with each trial condition (Fig. 4A) changed as the animals acquired the task (i.e., more object-in-place trial conditions and less response trial conditions identified in the later stage of learning), the correlation analyses performed above might have been influenced by an inequality in sampling across different trial conditions. To test this possibility, all correlation analyses (Fig. 4B) were performed again with bootstrap samples throughout the learning period (Ojakangas et al. 2006). For example, when ten trial samples were available for one condition (e.g., GC-left turn condition in arm 3) and three trial samples were available for another condition (e.g., GC-right turn condition in arm 3), bootstrap sampling was used to randomly sample three trials (out of 10 trials; resampling allowed) from the first condition and the same was done for the second condition. Then, the Pearson's $r$ was obtained between the two conditions. This procedure was repeated $1000 \times$ for each pair of trial conditions and the average coefficient was taken as $r_{\text {object-in-place }}$ or $r_{\text {response }}$ Therefore, if the original results were mainly driven by the trial condition with a larger number of trials, the parametric bootstrapping would negate the originally observed effects at the testing level of alpha $=0.001$. The resulting scatter plots and regression analysis results (both $P$-values $<$ 0.0001 ; slopes from the bootstrap analyses fell within 1 rootmean-squared-error of the slopes based on the raw data) were not significantly different from those presented in Figure 4B (Supplemental Fig. 5A). Furthermore, another bootstrapping analysis with shuffling method (Stark and Abeles 2005) was performed to test the possibility that the firing rate maps (especially the ones with a small sampling of trials) and resulting correlational results might be influenced by inadequate sampling. For this, the locations of pixels within a firing rate map were randomly shuffled within the rate map and the correlation coefficients were obtained based on the shuffled rate maps. This procedure was repeated $1000 \times$ for each pair of trials throughout the learning period. If the firing patterns obtained in the original data resulted by chance, the shuffling would not affect the coefficient, so the same result would be generated. No significant correlation was observed based on the method of shuffling in bootstrap (Supplemental Fig. 5B). Overall, all bootstrapping procedures suggest that correlations observed in Figure 4B were not obtained by chance or inadequate sampling.

It is unlikely that the relationships shown above were due to an increase in the running speed of the animals across training days. Although the running speed increased as the animals became familiarized with the task and as their performance increased $\left(r^{2}=0.33, P<0.0001\right.$, linear regression; Supplemental 
Fig. 6A), there was no significant relationship between the increase in running speed and the degree of similarity between rate maps for object-in-place trial conditions $\left(r^{2}=0.003, P>\right.$ 0.3; Supplemental Fig. 6B). Furthermore, whereas running speed continued to increase from Day 1 onward within the pre-learning period (significantly correlated with each other, $r^{2}=0.04, P<$ 0.05; Supplemental Fig. 6C), the rate map similarities between response-strategy trial conditions were not correlated with the training day $\left(r^{2}=0.001, P>0.8\right.$; Supplemental Fig. 6D) during that period.

\section{Emergence of object-in-place firing patterns at the ensemble level}

More detailed dynamics associated with the learning-related changes between pre- and post-learning periods were examined using ensembles of neurons (i.e., single units simultaneously recorded both within a subject and within a recording session) across the entire learning period (Fig. 5). In some representative examples shown in Figure 5 (only arm 3 data shown) from the animal's first day of training (D-6), neuronal ensembles fired mostly associated with the response-strategy-based trial conditions (see the two firing rate maps associated with the right-turn response for the neurons recorded on the first day, or D-6 of learning in Fig. 5) as the rat chose any object occupying the right food well. This stereotyped behavior resulted in chance-level performance (see the performance graph in Fig. 5). However, as the stereotyped response pattern decreased (see the performance graphs for GC and CG trials for rat 41 in Supplemental Fig. 1) toward the day (D0) in which the stepwise increase in performance occurred, the neurons simultaneously recorded in CA1 exhibited mixed firing patterns across different trial conditions. Specifically, most cells exhibited firing associated with object-in-place trial conditions (see firing rate maps in the first two columns for D-1 and D0 in Fig. 5) in addition to response-dependent trial conditions (firing rate maps between the first and the fourth columns for D-1 and D0 in Fig. 5). Importantly, however, the firing pattern correlated exclusively with the trial conditions for the response strategy (as in D-6) was never observed in this transitional period (examples shown for D-1 and D0 in Fig. 5). Compared to D-1, on the day (D0) when the performance of the rats jumped to $70 \%$ or more, neurons activated more strongly by object-in-place trials began to emerge (see tt 13 cell 4 and cell 5 in Fig. 5 for example) although mixed patterns of firing across trials associated with different strategies were still observed in other cells. As performance became stable toward the later phase of learning (e.g., $\mathrm{D}+3$ ), we were unable to observe cells that fired exclusively associated with response-strategy because most cells fired strongly in object-in-place trial conditions only. Although it was difficult to find more neuronal ensembles due to many conditional constraints (both behavioral and physiological), the qualitative illustration of the examples shown in Figure 5 demonstrates dynamically modulated firing rates in a given set of neurons in a single animal with changes in cognitive strategies.

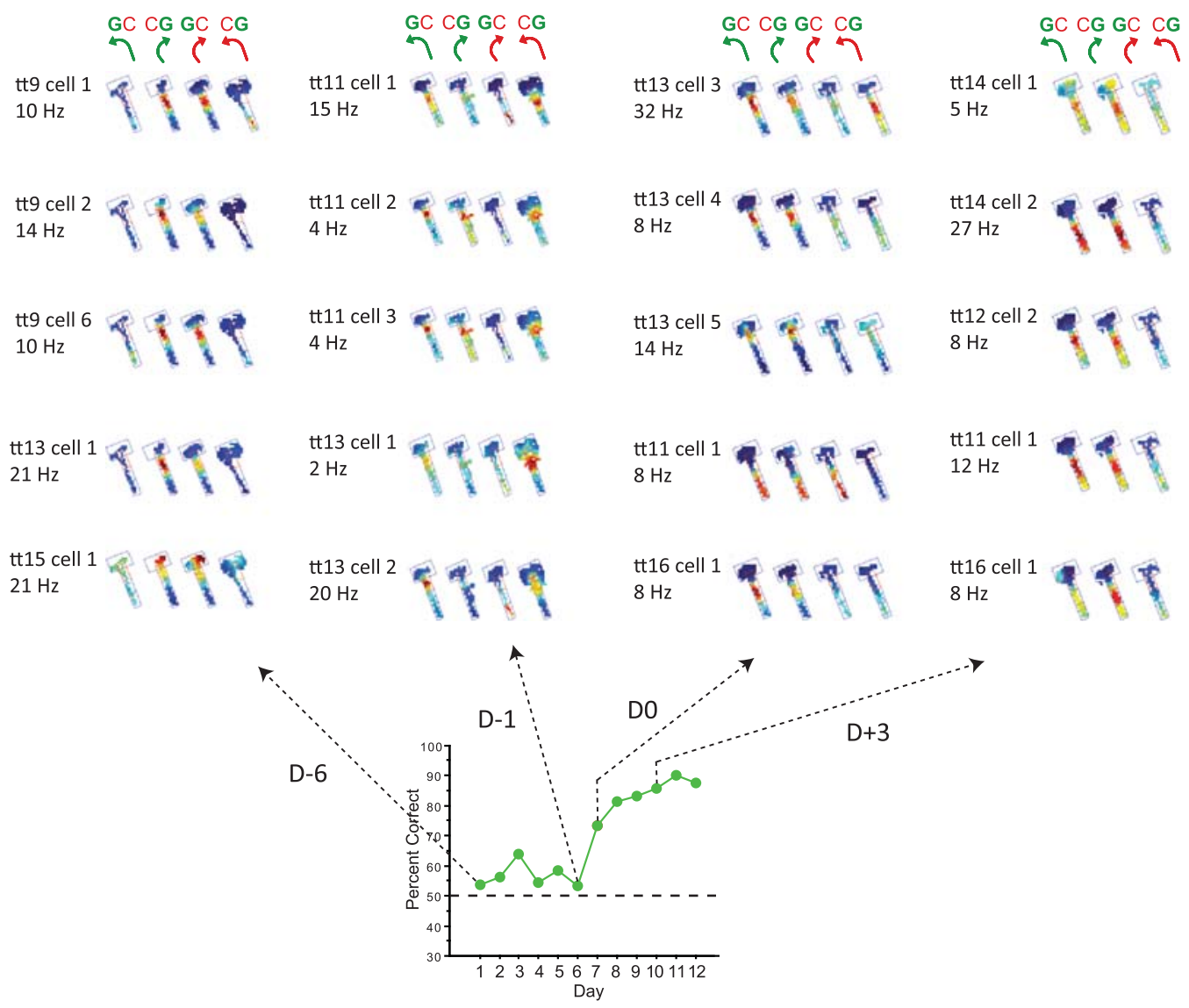

Figure 5. Ensemble firing patterns throughout learning in a single rat. Rate maps for different trial conditions from simultaneously recorded CA1 neurons are shown for selected days during learning. Maximal firing rate (hertz) is shown for each neuron. Line graphs show the overall performance (on average including arms 3 and 5) of the animal across days (upper) and the same animal's performances in arm 3 (middle) and arm 5 (lower) separately. Only trial conditions with over two trials/condition are shown. 


\section{Prospective neuronal firing increases after learning at the population level}

An interesting phenomenon was that strategy-related firing patterns were observed mostly before the rat reached the choice platform (thus, prospectively). Since the rats were able to see both objects from the moment they entered each arm in our task, an important issue is whether the hippocampal network was able to process upcoming reward-producing object-place events in a prospective manner (if so, in what area of the maze?). To address this issue, we examined which area of the arm underwent the biggest change in firing rate at the population level between object-in-place conditions (i.e., correct GC-left-turn trials and CG-right-turn trials) as the learning stage changed from prelearning to post-learning.

A population-vector correlation analysis was used for this purpose (Lee et al. 2004b) as follows. Firing patterns in different object configuration conditions (correct GC and correct CG) in the arm area were linearized and sorted according to the conditions. The linearized field ( $42 \mathrm{~cm}$ long) was produced for each object configuration condition in both arms (the number of trials $\geq 2$ as a minimum sampling requirement) per session. Twodimensional correlation coefficient was calculated between linear fields of the different object-in-place conditions in a given arm and comprised a population vector matrix. Each firing rate vector in the population matrix from one object-in-place condition (e.g., correct GC-left-turn) was cross-correlated with a matching firing rate vector from the other object-in-place condition (e.g., correct CG-right-turn) in the same arm to produce a correlation matrix. Overall, 250 pairs were used for the analysis $(n=88$ for prelearning and $n=162$ for post-learning). The population vector autocorrelation matrices between identical trial conditions are shown first to check if the population-vector correlation method works properly (left and middle columns in Fig. 6A). These autocorrelation matrices demonstrated a diagonal and perfect correlation band $\left(r^{2}=1\right)$ in both pre-learning and post-learning periods, as the same population vectors are correlated with each other. Interestingly, the correlation bands became wider especially in the proximal arm region during the post-learning period compared to the pre-learning period, implying that the firing field of a neuron was more extended in the proximal region of the arm under the same trial conditions as rats learned the task.

Comparing the population vectors between the corresponding zones in all regions (i.e., proximal and distal arm areas and the arm-choice platform joint region) revealed no significant differences ( $t$-test, $P$-values ranging from 0.06 to 0.98$)$. However, when the similarity in firing was calculated in the same arm between two different object configuration conditions associated with the object-in-place strategy, substantially increased levels of crosscorrelations were observed in the proximal arm region of the correlation matrix from the post-learning period $(P$-values ranging from 0.0001 to 0.03 in the $t$-test for population vectors) compared to the pre-learning period, whereas firing patterns were similar in other regions of the arm (Fig. 6A). The proximal portion of the arm was the start of different trajectories (or the entrance of the arm). Therefore, changes observed in the correlation matrix in this part of the arm strongly indicates that the hippocampal neurons started to respond to the object-in-place information viewed relatively farther away from the choice platform as the object-in-place learning took place. The correlation matrix for the pre-learning period, in contrast, demonstrates that the neurons did not fire similarly as the rats entered the arm. This is presumably because the animals were using the response strategy for which coding different future trajectories (left turn versus right turn) associated with different trial conditions (GC and CG) might be more important. For example, as some neurons
A
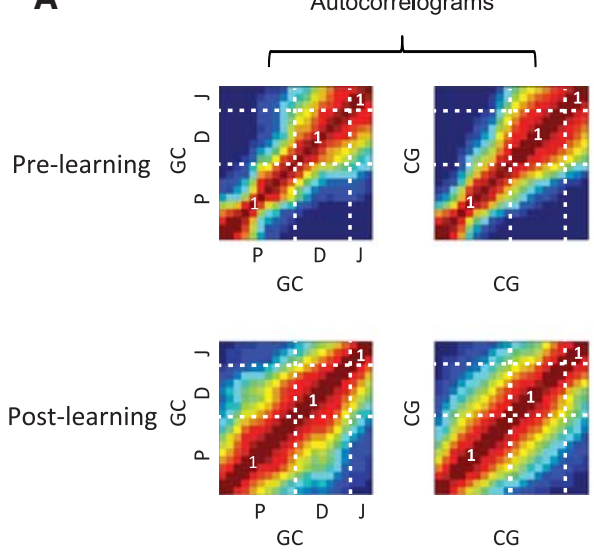

$B_{1}$

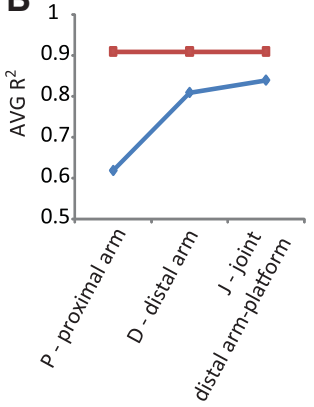

Correlograms b/w Object-in-Place trials

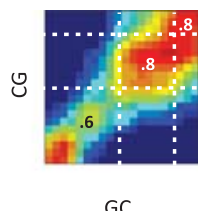

GC

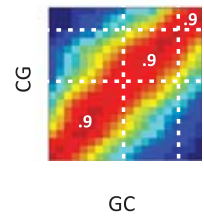

$\nrightarrow$ pre-learning - post-learning
Figure 6. Increase in the similarity between prospective neuronal firing after learning at a population level. $(A)$ Color-coded matrices showing correlations between population firing-rate vectors along the arm before and after the acquisition of the task (up to the junction between the distal arm and the choice platform, denoted by "J" in each matrix; " $\mathrm{P}$ " and " $\mathrm{D}$ " refer to the proximal and distal regions of the arm, respectively). "CG" and "GC" indicate trial types. Numbers in the matrix indicate correlation coefficients (along the diagonal) for different zones (the boundaries of which are marked by dotted lines) in the arm. (B) When pre- and post-learning periods were compared, only the proximal arm zone displayed a significantly increased similarity between object-in-place trial conditions. AVG R2 denotes the average correlation coefficient along the diagonal in a given zone (e.g., proximal arm or " $\mathrm{P}$ ") in the correlation matrix in $A$.

only tuned to the upcoming right turns associated with GC trials, these neurons will not fire very much in the prospective left-turn trials associated with CG trial conditions. This should bring the population correlation down to a lower level as shown in Figure 6A during pre-learning. Comparing average correlations along the diagonal also supported this observation (Fig. 6B). The population vector correlation analysis thus reveals that the emergence of similar firing patterns at the prospective portion of the arm between the trial conditions may serve as a critical neurobehavioral indicator for the acquisition of the object-place paired-associate task.

\section{Discussion}

The current study examined changes in neural firing patterns in the hippocampus during the object-place paired-associate task in rats. In the task, the initially dominant, directional bias must be inhibited and a shift to an object-in-place strategy must occur during learning. We observed a crossover between these two strategies within subjects during learning along with the matching changes in neural firing patterns in a prospective manner. These results provide compelling evidence that the firing patterns of 
hippocampal neurons are heavily influenced, yet flexibly changed by the dominant strategy at a given learning stage.

Prior studies have shown that hippocampal neurons can fire differentially according to different spatial tasks in the same environment (Markus et al. 1995; Gothard et al. 1996a,b; Wood et al. 2000; Ferbinteanu and Shapiro 2003; Lee et al. 2006). The tasks frequently used in those studies include random or directed food search and spatial alternation using open platforms and/or linear tracks. Those studies demonstrated that neuronal firing in the hippocampus is associated with different frames of reference in a given task and the reference frame is defined by egocentric, allocentric, and/or navigational sequence information. In some studies (Wood et al. 2000; Ferbinteanu and Shapiro 2003; Lee et al. 2006), neuronal firing in the hippocampus was bound to a certain location (e.g., a particular location of a track) but the activity of the neuron was modulated by task demand. It has been proposed that this type of rate modulation within a fixed spatial receptive field may serve as a hippocampal mechanism for representing episodic memory composed of a location and events occurring in that location (Leutgeb et al. 2005). However, considering that an event or episode in an animal's environment is often composed of both spatial and nonspatial information, our study addresses this possibility more sufficiently because spatial information (i.e., arm) needs to be processed accurately as nonspatial information (i.e., object) needs to be associated with the spatial information. The results from our study corroborate the results from prior studies. Although most neurons represented specific arm information (Figs. 2A and C), and exhibited a well-formed place field, cells tended to fire more strongly in correlation with the rat's prospective choice behavior (i.e., response bias) during the pre-learning stage. However, during the post-learning stage, cells tended to fire more strongly in correlation with the rat's prospective choice behavior targeted to the correct object regardless of the turning direction associated with the choice of the object. Since the same objects (and other room cues) were always present throughout the learning process, these results demonstrate that it is the animal's internal variable (such as task demand or strategy for associating a certain object with space) that changes the level of activity in hippocampal neurons in our task.

The results of our study extend previous findings by testing novel components as follows. First, neural activity throughout the entire acquisition period was examined. In contrast to the fact that most prior studies overtrained animals before recordings were made, the animals in our study never learned the objectplace paired-associate task before actual recordings took place. Second, when changes in rules or strategies were introduced in previous studies (Markus et al. 1995; Ferbinteanu and Shapiro 2003; Smith and Mizumori 2006), those changes were imposed by experimenters. On the contrary, two naturally competing strategies (i.e., response and object-in-place) existed in our task and the animals must switch from one strategy to the other during the normal course of learning. Third, because objects were always approached from the same entrance (as opposed to a plus maze or continuous T-maze) in a unidirectional manner, the prospective firing pattern exhibited in the arm can be interpreted without causing confusion in the temporal reference frame (such as the possibility of retrospective firing).

As shown in various selectivity indices in Figure 2C, the only strong firing correlate with the global task structure (e.g., across different trials and conditions) was the arm-specific firing pattern. We rarely observed a neuron that showed a good place field in one arm and that fired in the other arm with the same magnitude at the same time. In contrast, neuronal activity was not specifically tied to a particular object across different arms and conditions, and it was not associated with a particular object configuration either. CA1 neurons did not represent a particular direction of turning throughout the task. These results in our study overall suggest that, in the hippocampus, spatial information (e.g., arm) is likely to serve as the strongest reference frame within which nonspatial information is organized. For example, the modulation of firing rates associated with different object-place paired-associative conditions occurred within the overall place field of a neuron in our study. This may be somewhat different from the results from a previous physiological study (Rolls et al. 2005) where the presence of object-representing neurons in CA1 (as well as in CA3) was reported. Because anatomical studies (Witter 1993) suggest that relatively nonspatial LEC and spatial MEC inputs are more segregated along the proximal and distal cell layers in CA1, it may still be possible to observe object-representing neurons in CA1 in some conditions if more proper anatomical subfields are targeted strategically. On the basis of the results from the current study, however, it appears that object information is always represented in conjunction with spatial information in the hippocampus in rats.

When the rat ran toward an object on its particular side (e.g., left side) throughout the session during the earlier period of learning in our study, we operationally defined such behavior as response bias because such a response was not associated with object identity (but seemingly with the turning direction only). On the other hand, it is also possible that the animal might have chosen a particular location (e.g., left food well) instead of using the egocentric strategy. However, when the directional turning bias was graphed for individual rats (Supplemental Fig. 7) for the earlier learning period (Days 1-3) in which response bias was maximal, rat 21 showed left-turn bias in both arms and rat 24 showed right-turn bias in both arms. This indicates that it is more likely that the two rats were using a simple turning response strategy. Rat 41 , in contrast, showed left-turn bias in arm 3 and right-turn bias in arm 5. One may argue that the rat was not using the response strategy during Days 1-2 because it did not turn to the same side in different arms. However, it is equally possible that this may be that the rat may have associated a particular turning bias with a specific arm. Although it will never be possible to determine whether the animals used exclusively response bias without considering any place variable (e.g., food well location in the room), given that two rats out of three showed response biases associated with particular turning directions regardless of arm locations, it would be reasonable to label such a behavioral pattern as response bias.

Regarding the time course of the appearance of egocentric versus allocentric strategies, it would be fair to point out that the task used in the current study is not a simple spatial navigation task that has been widely used to test the allocentric versus egocentric strategy issue (Packard et al. 1989; Packard and McGaugh 1996; Chang and Gold 2003; Lee et al. 2008). Instead, in the current task, the animals were required in addition to associate objects with allocentric spatial cues. Furthermore, the biconditional component of the task made the task more difficult because both object and spatial variables needed to be considered at the same time to result in reward. Therefore, one can see that the response strategy would be a much simpler strategy to be tested compared to the object-in-place strategy during the earlier phase of learning. In contrast, in a traditional T-maze task, for example, allocentric versus egocentric strategies may compete more equally from the beginning and the allocentric strategy may win out in the earlier learning stage in such an occasion as the literature suggests (Packard and McGaugh 1996; Chang and Gold 2003).

Prior studies have shown that the firing location associated with the place cell in the hippocampus shifts toward a significant location in the environment with respect to reward delivery and goal-related navigation pattern (Breese et al. 1989; Markus et al. 1995; Kobayashi et al. 1997; Fyhn et al. 2002; Lee et al. 2006). 
In our study, only $26 \%$ of spatial firing fields were located in the proximal portion of the arms on average and $74 \%$ of firing fields were located in the distal portion and choice platform in the arm (Fig. 2B). The inhomogeneous distribution of spatial firing fields strongly suggests that hippocampal neurons do not map different spatial locations equally. It is conceivable that the distal portion-to-choice platform is a more important area to the animals than the proximal portion of the arm because the expectation of reward and its associated response should be made as the rats approach the choice platform. Visual identification of objects may occur better near the choice platform too. There were almost equal proportions of neuronal firing fields in the choice point (where the distal arm meets the choice platform) and in the proximal portion of the arm (20\% in the choice point and $26 \%$ in the proximal region of the arm), which further suggests that the significance of spatial location (defined by task demand) heavily influences where the cells fire in the hippocampus.

A leading theoretical framework based on anatomical and physiological studies suggests fairly segregated paths for information processing in the upstream regions of the hippocampus (Quirk et al. 1992; Burwell 2000; Witter et al. 2000; Fyhn et al. 2004; Hargreaves et al. 2005; Knierim et al. 2006; van Strien et al. 2009). We have shown that the perirhinal cortex is essential for this task (presumably providing object information), especially when rats need to learn new object-place paired associations (Jo and Lee 2010). In this theoretical framework, it is considered that the hippocampus is the center where the segregated, spatial versus nonspatial inputs are associated together to form a representation for an event. Since nonspatial and spatial inputs are fed to the hippocampus at the same time, whether nonspatial information such as the identity of an object may exist totally independent of spatial information in the hippocampus begs the question. The results from our study suggests that nonspatial information in the hippocampus does not exist independent of the spatial variables associated with it (at least in CA1), but can modulate spatial firing of neurons powerfully as objects must be associated with different locations in the environment. It will be interesting in the future to examine whether other subfields (e.g., CA3 and dentate gyrus [DG]) of the hippocampus and its associated cortical regions show differential firing properties in such situations where discrete events are defined by unique combinations of spatial and nonspatial variables in the environment. In addition, Lee and Solivan (2010) have recently demonstrated that the DG is important in the current task presumably by providing orthogonal spatial representations associated with different arms and object locations. Recording single units from DG in the current study in the future may show the development of more orthogonal spatial firing patterns, for example, between arm 3 and arm 5 as rats acquire the task. Although the role of CA3 has not been tested, the involvement of DG in the current task suggests that it is highly likely that CA3 is also important in the task. The CA3 may be in charge of rapid and arbitrary associations between nonspatial information (i.e., objects) and spatial information in the current task. In such cases, it is expected that CA3 may show the development of an object-place paired- associative representation earlier than DG and CA1.

It was originally reported that episodic memory was impaired in amnesic patients with hippocampal damage (Scoville and Milner 1957), and testing the capability of animals in associating individual objects with different places (or background visual scenes) has been considered as an essential animal model (Gaffan 1994; Gilbert and Kesner 2002; Wirth et al. 2003; Rolls et al. 2005; Lee and Solivan 2008). However, only a limited number of physiological studies have recorded hippocampal neurons in a task that uses object-place associative rules explicitly (Wirth et al. 2003; Rolls et al. 2005; Komorowski et al. 2009). Our results show that hippocampal neurons exhibit robust physiological correlates for object-place associative learning in a prospective manner when the discrimination of objects is possible from a distance. This may be similar to a primate study in which hippocampal neurons fired in correlation with specific views of an environment (Rolls et al. 1997). It is possible that similar firing properties can be assessed in rodents if the task requires judging the reward contingency of an event remotely using their vision as in the current task.

\section{Materials and Methods}

\section{Subjects}

Three male Long-Evans rats (300-400 g) were used in the study. Once delivered, all animals were kept in an animal colony for a week for acclimation before being used in the study. All animals were maintained on a 12-h/12-h light/dark cycle and behavioral experiments were performed in the light phase of the cycle. The rats were housed individually and were maintained at $85 \%$ of their free-feeding weight (with water provided ad libitum) to facilitate motivation for the behavioral task. Protocols for animal care and surgery followed the guidelines of the National Institutes of Health and the Institutional Animal Care and Use Committee.

\section{Handling and shaping}

Once acclimated to the environment, rats were tamed and handled by an experimenter. As part of the handling, they were allowed to freely forage for $1 / 8$ pieces of sugar-coated cereal scattered in a rectangular arena outside the behavioral testing room. Once the animals showed signs of taming (e.g., no defecation or urination) and eagerly consumed the cereal reward, a shaping procedure was used to train the rats to displace an object. For this purpose, a rectangular platform (identical with the "choice platform" used in the radial maze in the behavioral recording room) made of black Plexiglas $(23 \times 30 \mathrm{~cm})$ was placed in a random location each day in the rectangular arena. Three food wells (each $2.5 \mathrm{~cm}$ in diameter and $0.6 \mathrm{~cm}$ deep; separated from each other by $17 \mathrm{~cm}$ ) were present in the platform. An $1 / 8$ piece of cereal reward was placed in one of the food wells (randomly chosen) and a black wooden block attached to a heavy washer gradually covered the food well until the rats learned to displace the block to find the cereal reward. Once the animals learned this object-displacing behavior, they were given implantation surgery for recording devices.

\section{Surgery and implantation of a microdrive array}

Each animal was initially placed in an induction chamber for isoflurane gas anesthesia ( $4 \%$ isoflurane with $100 \% \mathrm{O}_{2}$ for $2-3 \mathrm{~min}$ ) and, once immobile, was injected with ketamine $(55 \mathrm{mg} / \mathrm{kg})$ and xylazine $(6 \mathrm{mg} / \mathrm{kg})$. The rat was then placed in a stereotaxic frame and its head was fixed in the frame. Anesthesia was maintained throughout surgery by isoflurane inhalation $(0.5 \%-2 \%$ isoflurane with $100 \% \mathrm{O}_{2}$ ). A custom-made recording device (microdrive array or "hyperdrive") with tetrodes was implanted. Tetrodes were made by twisting four nichrome wires (each wire $12 \mu \mathrm{m}$ in diameter; Kanthal). The final impedance for each wire was adjusted to $150-300 \mathrm{k} \Omega$ (measured in gold solution at $1 \mathrm{kHz}$ with an impedance tester, IMP-1; BAK electronics) before implantation. Eight tetrodes and one reference wire were implanted in the right dorsal hippocampus ( $3.0 \mathrm{~mm}$ posterior to bregma and $1.7 \mathrm{~mm}$ lateral from midline). An insulated stainless-steel wire connected the electrode-interface board of the hyperdrive to a ground screw in the skull.

\section{Apparatus and testing environment}

An elevated, radial arm maze made of black Plexiglas (Fig. 1A) was used throughout the experiment. The maze was composed of seven radial arms (each $8 \times 80 \mathrm{~cm}$, separated by $25.7^{\circ}$ from each 
other). The arms radiated from a circular center stage $(48 \mathrm{~cm}$ in diameter). The distal end of each arm was connected to a rectangular platform $(23 \times 30 \mathrm{~cm}$; choice platform as shown in Fig. 1A insets) in which objects were presented. Each choice platform contained three food wells and at the bottom of each food well, a set of infrared emitter and detector was installed to detect the displacement of an object instantly. The infrared detector sent TTL signals to a data acquisition system (Digital Lynx, Neuralynx) and the timing for the object displacement was recorded with neural signals. Food wells were separated from each other by transparent vertical dividers made of Plexiglas (each $6 \times 13 \mathrm{~cm})$. The transparent dividers were placed to encourage a more explicit and targeted response to a discrete food well. A transparent guillotine door $(10 \times 25 \mathrm{~cm})$ was present at the entrance of each arm to control the animal's access to the arm. In the entrance region of each arm, transparent Plexiglas walls (each $25 \times 13 \mathrm{~cm}$ ) prevented the animals from jumping to adjacent arms in the maze. A start box $(20 \times 25 \times 30 \mathrm{~cm})$ with a black and opaque guillotine door was located in the center stage. The maze was placed in the center of a circular curtained area (260 $\mathrm{cm}$ in diameter) decorated with distinctive, hanging visual cues. A CCD camera, dimmable halogen light, and commutator were positioned above the maze and two loud speakers were placed underneath the center stage of the maze to provide white noise during behavioral experiments.

\section{Behavioral training}

Once the animals recovered from surgery, they were trained in a biconditional object-place paired-associate task (Fig. 1A). Detailed descriptions of training procedures were provided in a previous study (Lee and Solivan 2008). Briefly, before each trial began, the rat was placed in the start box with the guillotine door closed. Once the experimenter opened the guillotine door, the animal came out and entered an arm that was open for that trial. Only arm 3 and arm 5 (Fig. 1A) were used throughout the task. In the choice platform at the end of the arm, the animal had to choose between two objects (a toy girl and a cylinder). Displacing one of the objects resulted in either a cereal reward in the food well or no reward depending on the object-place paired-associative rule tied to the arm; specifically, the toy girl, but not the cylinder, was rewarded in arm 3 and vice versa in arm 5. When the rat made the correct choice, the rat was allowed to grab the cereal reward and the experimenter gently guided the animal to the start box so that the food reward was consumed in the start box. Rats quickly learned this procedure and voluntarily returned to the start box. In contrast, when the rat made an incorrect choice, the animal was prevented from choosing an alternate object (by a small Plexiglas panel in the experimenter's hand) and was gently guided to the start box. Objects were only available in the arm that was visited in a given trial and no object was available in the other arm during that time. This was to make sure that the neural firing correlates with objects were clearly associated with the objects presented in a given arm instead of the ones presented in other places in the maze. Once the trial ended, the rat was left in the start box until the experimenter prepared the choice platform for the next trial with the objects and reward. A typical intertrial interval was approximately $20-30 \mathrm{sec}$. Sixty-four trials were run per training session and a single training session was given per day.

\section{Single-unit recording and position tracking}

Behavioral training began after recovery from surgery, and tetrodes were lowered over several days while the rats slept in a custom-made recording booth outside the behavioral testing room. The electrode interface board (EIB) of the hyperdrive was connected to a unity-gain preamplifier (Neuralynx) and neural data were transferred to the digital Lynx acquisition system (32 kHz sampling rate; Neuralynx). Spiking signals from each tetrode were amplified 1000-10,000 $\times$ and band-pass filtered at $600-6000 \mathrm{~Hz}$. Continuous EEG signals from the same tetrode were amplified 2000-5000 $\times$ and band-pass filtered at
$1-1000 \mathrm{~Hz}$. In the behavioral recording room, the signals were delivered to the acquisition system outside the testing room through a slip-ring commutator (Neuralynx). For tracking the position of the animal, a custom-built LED array (composed of red and green LEDs) was connected to the EIB. The LED signals were captured by a digital camera placed in the ceiling and was fed to the acquisition system simultaneously via a video timestamp titler (sampling rate $=30 \mathrm{~Hz}$ ) and a digital video recorder. The whole maze arm area was recorded with $640 \times 480$ pixel resolution with a single pixel representing $0.09 \mathrm{~cm}^{2}$.

\section{Histological verification of recording sites}

Tetrode locations were verified after the completion of experiments as described elsewhere (Lee et al. 2004a,b). Briefly, after marker lesions were made electrolytically for individual tetrodes, the rats were perfused transcardially and the brain was cut at $30 \mu \mathrm{m}$ thickness using a freezing sliding microtome. Cut sections were stained with thionin and digital photomicrographs of the sections were taken. The serial photomicrographs were then used to reconstruct each tetrode's trace in the brain. Physiological recording data and depth profile were also used along with the histological data.

\section{Unit isolation and screening}

Unit isolation was conducted following the procedures described in detail elsewhere (Lee et al. 2004a,b, 2006). Briefly, single units were isolated offline using custom software running on a Windows-based PC. Single units were isolated by comparing the signals recorded in four wires of a tetrode and multiple parameters associated with spike waveforms such as peak (the main parameter), width, height, energy, etc., were used in the process. In addition, only neurons showing complex-spike bursts identified in the signature interspike interval histogram of hippocampal complex-spike neurons (Fox and Ranck 1981; Lee et al. 2006) were used in the analysis. To further ensure that complex-spike units, but not theta cells, were analyzed in the current study, the spatial information score (Skaggs et al. 1993) was calculated for units that generated $\geq 100$ spikes during outbound journeys within a single recording session and, among those, only units that exhibited an information score of $0.5(P<0.01)$ or above in the maze were further analyzed. For each behavioral recording session, units showing poor stability between the pre- and post-sleep recording sessions (Lee et al. 2004a,b) were not included in the analysis.

Units that had firing fields representing the start box or the center platform were not analyzed in the current study mainly due to inhomogeneous position-sampling issues (i.e., rats visited only limited locations in those places, thus limiting the interpretation of the firing patterns in those areas). To determine the location of firing, the center-of-mass (COM) of the overall 2-D firing field in the recording session was calculated (Fenton et al. 2000; Lee et al. 2004) for each unit and whether to exclude the field was determined on the basis of whether the COM of a field was in the start box or center platform. Units whose firing fields started from the center platform and extended up to only the entrance region ( $15 \mathrm{~cm}$ from the arm entrance) of each arm were also excluded because this region was partitioned by clear Plexiglas walls on both sides and rats tended to stay stationary in that area during the earlier period of training. Among the units that fired in the arm and/or choice platform, a unit was considered to have its spatial firing field once at least 100 spikes were generated in those regions and its average firing rate was equal to or above $1 \mathrm{~Hz}$. The arm plus the choice platform regions were divided into four zones for localization of spatial firing fields of neurons as follows: choice platform - the rectangular platform area (excluding the joint area between the distal arm and the platform); arm-choice platform junction-the joint area where the distal end of the arm met the choice platform; distal arm$20 \mathrm{~cm}$ from the upper end of the arm; proximal arm $-22 \mathrm{~cm}$ from the lower boundary of the distal arm zone (Fig. 2B). 


\section{Data analysis}

\section{Analysis of spatial firing pattern}

The area in which the maze was positioned in the behavioral testing room was represented by a $64 \times 48$ matrix for constructing 2-D spatial firing rate maps ( 1 pixel square represents $0.09 \mathrm{~cm}^{2}$ ). The 2-D rate map for a single unit in the maze was constructed by dividing the number of spikes generated by a neuron in a given bin by the duration of stay in seconds in that bin. Only bins visited during the behavioral recording session were included in the analysis. An adaptive binning algorithm was used to balance sampling error and spatial resolution (Skaggs et al. 1996). An outbound journey was defined as the period from the moment the rat entered the arm (measured by detecting the crossing of position data across an imaginary line) until one of the objects was pushed in the choice platform (detected by an infrared sensor underneath each food well). An inbound journey was defined as the period from the end of the outbound journey to exiting the arm (i.e., re-entering the center platform and the start box). Only outbound journeys were used for analysis in this study mainly because behaviors of the rats during inbound journeys (especially immediately after pushing the first object in the choice platform) were variable between trials. For example, sometimes rats were guided or blocked by the experimenter with a small Plexiglas panel when they refused to come back to the start box or they tried to correct a wrong choice by choosing another object.

\section{Selectivity index calculation}

To test if neural firing patterns were correlated with single factors in the task, various selectivity indices were calculated as follows. An arm-selectivity index calculated how specifically the firing of a neuron was correlated with a certain arm (between arm 3 and arm 5) as follows. Arm-selectivity index $=\mid($ FR[arm3] FR[arm5]) / (FR[arm3] + FR[arm5])|, where FR(armX) stands for the average firing rate (hertz) in the arm 3 or 5. For example, FR(arm3) includes all trials in which the rat visited arm 3 regardless of its object choices in that arm (thus involving both correct and incorrect choices). An object-selectivity index calculated how specifically neuronal firing was tied to the choice of a certain object (e.g., toy girl) regardless of other information (i.e., the arm where the object appears and the left or right turn associated with the object choice). Object-selectivity index $=\mid(\mathrm{FR}[\mathrm{G}]-\mathrm{FR}[\mathrm{C}]) /$ $(\mathrm{FR}[\mathrm{G}]+\mathrm{FR}[\mathrm{C}]) \mid$, where $\mathrm{FR}(\mathrm{X})$ denotes the average firing rate (hertz) of a neuron in all the trials in which a particular object $\mathrm{X}$ (G: toy girl, C: cylinder) was chosen. A response-selectivity index measured the degree to which neuronal firing was associated with either particular turning direction within a choice platform. Response-selectivity index $=|(\mathrm{FR}[\mathrm{L}]-\mathrm{FR}[\mathrm{R}]) /(\mathrm{FR}[\mathrm{L}]+\mathrm{FR}[\mathrm{R}])|$, where $\mathrm{FR}(\mathrm{L})$ indicates the average firing rate of a neuron in the trials in which the animal chose an object in the left food well (regardless of object identity and arm information) and likewise for FR(R). An object configuration-selectivity index was also calculated in a similar fashion between GC and CG trial conditions (GC indicating the toy girl to the left and cylinder to the right, and vice versa for $C G)$.

\section{Cross-correlation between 2-D rate maps}

The similarity of spatial firing patterns of a neuron between different conditions of trials was measured by calculating the Pearson's correlation coefficient $\left(r^{2}\right)$ between the rate maps (pixel-by-pixel) separately constructed for those conditions. The pixels that were not visited in both conditions were not included in this process. Each maze arm was narrowly designed $(8 \mathrm{~cm})$ to maximize trajectory overlap, and cross-correlation analyses throughout the study were restricted only to the areas with overlapping trajectories (resulting in the exclusion of the choice platforms and center platform). For Fisher's r-to-z transformation, the correlation coefficients were subject to the following formula: Z-score $=1 / 2 \times \ln$ $[(1+r) /(1-r)]$.

A firing field in the arm was represented as a linear field. Specifically, each arm was divided into 30 bins and the number of spikes fired in each bin was divided by the duration of stay (seconds) in that bin (included were only spikes collected when the rat's running speeds were at least $5 \mathrm{~cm} / \mathrm{sec}$ ). The resulting firing-rate histogram was smoothed with a Gaussian kernel (full width at half-maximum at 2). The boundaries marking the start and end of a firing field were determined by identifying the bins at which the firing rates dropped below $10 \%$ of the maximum firing rate of the linearized field. A population vector correlation analysis was performed as described previously (Lee et al. 2004).

\section{Acknowledgments}

The current study was supported by the WCU program of the Ministry of Education, Science and Technology in Korea through KOSEF (R32-10142) and by NIMH (R01 MH079971). We thank Dmytro Nikolayenko for his assistance in data collection and analysis.

\section{References}

Breese CR, Hampson RE, Deadwyler SA. 1989. Hippocampal place cells: Stereotypy and plasticity. J Neurosci 9: 1097-1111.

Burwell RD. 2000. The parahippocampal region: Corticocortical connectivity. Ann N Y Acad Sci 911: 25-42.

Chang Q, Gold PE. 2003. Switching memory systems during learning: Changes in patterns of brain acetylcholine release in the hippocampus and striatum in rats. J Neurosci 23: 3001-3005.

Eifuku S, Nishijo H, Kita T, Ono T. 1995. Neuronal activity in the primate hippocampal formation during a conditional association task based on the subject's location. J Neurosci 15: 4952-4969.

Eschenko O, Mizumori SJY. 2007. Memory influences on hippocampal and striatal neural codes: Effects of a shift between task rules. Neurobiol Learn Mem 87: 495-509.

Fenton AA, Csizmadia G, Muller RU. 2000. Conjoint control of hippocampal place cell firing by two visual stimuli. Ii. A vector-field theory that predicts modifications of the representation of the environment. J Gen Physiol 116: 211-221.

Ferbinteanu J, Shapiro ML. 2003. Prospective and retrospective memory coding in the hippocampus. Neuron 40: 1227-1239.

Fox SE, Ranck JB Jr. 1981. Electrophysiological characteristics of hippocampal complex-spike cells and theta cells. Exp Brain Res 41: 399-410.

Fyhn M, Molden S, Hollup S, Moser MB, Moser E. 2002. Hippocampal neurons responding to first-time dislocation of a target object. Neuron 35: $555-566$.

Fyhn M, Molden S, Witter MP, Moser EI, Moser MB. 2004. Spatial representation in the entorhinal cortex. Science 305: 1258-1264.

Gaffan D. 1994. Scene-specific memory for objects: A model of episodic memory impairment in monkeys with fornix transection. J Cogn Neurosci 6: $305-320$.

Gilbert PE, Kesner RP. 2002. Role of the rodent hippocampus in paired-associate learning involving associations between a stimulus and a spatial location. Behav Neurosci 116: 63-71.

Gothard KM, Skaggs WE, McNaughton BL. 1996a. Dynamics of mismatch correction in the hippocampal ensemble code for space: Interaction between path integration and environmental cues. J Neurosci 16: $8027-8040$.

Gothard KM, Skaggs WE, Moore KM, McNaughton BL. 1996b. Binding of hippocampal CA1 neural activity to multiple reference frames in a landmark-based navigation task. J Neurosci 16: 823-835.

Hargreaves EL, Rao G, Lee I, Knierim JJ. 2005. Major dissociation between medial and lateral entorhinal input to dorsal hippocampus. Science 308: $1792-1794$

Jo YS, Lee I. 2010. Perirhinal cortex is necessary for acquiring, but not for retrieving object-place paired association. Learn Mem 17: 97-103.

Jung MW, Wiener SI, McNaughton BL. 1994. Comparison of spatial firing characteristics of units in dorsal and ventral hippocampus of the rat. J Neurosci 14: 7347-7356.

Knierim JJ, Lee I, Hargreaves EL. 2006. Hippocampal place cells: Parallel input streams, subregional processing, and implications for episodic memory. Hippocampus 16: 755-764.

Kobayashi T, Nishijo H, Fukuda M, Bures J, Ono T. 1997. Task-dependent representations in rat hippocampal place neurons. J Neurophysiol 78: $597-613$.

Komorowski RW, Manns JR, Eichenbaum H. 2009. Robust conjunctive item-place coding by hippocampal neurons parallels learning what happens where. J Neurosci 29: 9918-9929. 
Lee I, Solivan F. 2008. The roles of the medial prefrontal cortex and hippocampus in a spatial paired-association task. Learn Mem 15: 357-367.

Lee I, Solivan F. 2010. Dentate gyrus is necessary for disambiguating similar object-place representations. Learn Mem 17: 252-258.

Lee I, Rao G, Knierim JJ. 2004a. A double dissociation between hippocampal subfields: Differential time course of CA3 and CA1 place cells for processing changed environments. Neuron 42: 803-815.

Lee I, Yoganarasimha D, Rao G, Knierim JJ. 2004b. Comparison of population coherence of place cells in hippocampal subfields CA1 and CA3. Nature 430: 456-459.

Lee I, Griffin AL, Zilli EA, Eichenbaum H, Hasselmo ME. 2006. Gradual translocation of spatial correlates of neuronal firing in the hippocampus toward prospective reward locations. Neuron 51: 639-650.

Lee AS, Duman RS, Pittenger C. 2008. A double dissociation revealing bidirectional competition between striatum and hippocampus during learning. Proc Natl Acad Sci U S A 105: 17163-17168.

Leutgeb S, Leutgeb JK, Barnes CA, Moser EI, McNaughton BL, Moser MB. 2005. Independent codes for spatial and episodic memory in hippocampal neuronal ensembles. Science 309: 619-623.

Markus EJ, Qin YL, Leonard B, Skaggs WE, McNaughton BL, Barnes CA 1995. Interactions between location and task affect the spatial and directional firing of hippocampal neurons. J Neurosci 15: 7079-7094.

Muller RU, Kubie JL, Ranck JB Jr. 1987. Spatial firing patterns of hippocampal complex-spike cells in a fixed environment. J Neurosci 7: 1935-1950.

Ojakangas CL, Shaikhouni A, Friehs GM, Caplan AH, Serruya MD, Saleh M, Morris DS, Donoghue JP. 2006. Decoding movement intent from human premotor cortex neurons for neural prosthetic applications. J Clin Neurophysiol 23: $577-584$.

O'Keefe J, Dostrovsky J. 1971. The hippocampus as a spatial map: Preliminary evidence from unit activity in the freely moving rat. Brain Res 34: 171-175.

O'Reilly RC, McClelland JL. 1994. Hippocampal conjunctive encoding, storage, and recall: Avoiding a trade-off. Hippocampus 4: 661-682.

Packard MG, McGaugh JL. 1996. Inactivation of hippocampus or caudate nucleus with lidocaine differentially affects expression of place and response learning. Neurobiol Learn Mem 65: 65-72.

Packard M, Hirsh R, White N. 1989. Differential effects of fornix and caudate nucleus lesions on two radial maze tasks: Evidence for multiple memory systems. J Neurosci 9: 1465-1472.
Poldrack RA, Packard MG. 2003. Competition among multiple memory systems: Converging evidence from animal and human brain studies. Neuropsychologia 41: $245-251$.

Quirk GJ, Muller RU, Kubie JL, Ranck JB Jr. 1992. The positional firing properties of medial entorhinal neurons: Description and comparison with hippocampal place cells. J Neurosci 12: 1945-1963.

Rolls ET, Robertson RG, Georges-Francois P. 1997. Spatial view cells in the primate hippocampus. Eur J Neurosci 9: 1789-1794.

Rolls ET, Xiang J, Franco L. 2005. Object, space, and object-space representations in the primate hippocampus. J Neurophysiol 94: $833-844$.

Scoville WB, Milner B. 1957. Loss of recent memory after bilateral hippocampal lesions. J Neurol Neurosurg Psychiatry 20: 11-21.

Skaggs WE, McNaughton BL, Gothard KM, Markus EJ. 1993. An information-theoretic approach to deciphering the hippocampal code. In Advances in neural information processing systems (ed. SJ Hanson, JD Cowan, CL Giles), pp. 1030-1037. Morgan Kaufman, San Mateo, CA

Skaggs WE, McNaughton BL, Wilson MA, Barnes CA. 1996. Theta phase precession in hippocampal neuronal populations and the compression of temporal sequences. Hippocampus 6: 149-172.

Smith DM, Mizumori SJ. 2006. Learning-related development of context-specific neuronal responses to places and events: The hippocampal role in context processing. J Neurosci 26: 3154-3163.

Stark E, Abeles M. 2005. Applying resampling methods to neurophysiological data. J Neurosci Methods 145: 133-144.

van Strien NM, Cappaert NLM, Witter MP. 2009. The anatomy of memory: An interactive overview of the parahippocampal-hippocampal network. Nat Rev Neurosci 10: 272-282.

Wirth S, Yanike M, Frank LM, Smith AC, Brown EN, Suzuki WA. 2003. Single neurons in the monkey hippocampus and learning of new associations. Science 300: 1578-1581.

Witter MP. 1993. Organization of the entorhinal-hippocampal system: A review of current anatomical data. Hippocampus 3: 33-44.

Witter MP, Wouterlood FG, Naber PA, Haeften TV. 2000. Anatomical organization of the parahippocampal-hippocampal network. Ann N Y Acad Sci 911: 1-24.

Wood ER, Dudchenko PA, Robitsek RJ, Eichenbaum H. 2000. Hippocampal neurons encode information about different types of memory episodes occurring in the same location. Neuron 27: 623-633.

Received April 8, 2010; accepted in revised form June 10, 2010. 


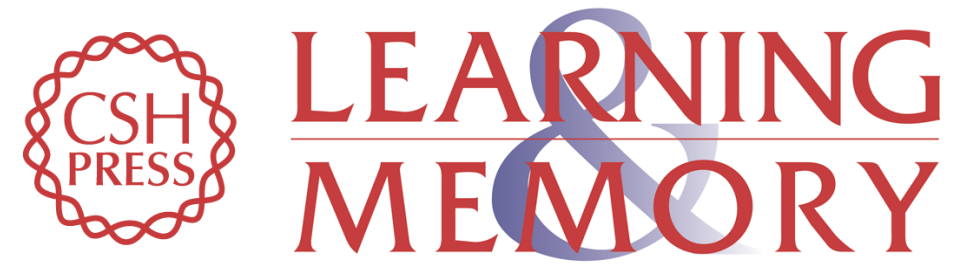

\section{The shift from a response strategy to object-in-place strategy during learning is accompanied by a matching shift in neural firing correlates in the hippocampus}

Inah Lee and Jangjin Kim

Learn. Mem. 2010, 17:

Access the most recent version at doi:10.1101/lm.1829110

Supplemental http://learnmem.cshlp.org/content/suppl/2010/07/29/17.8.381.DC1

Material

References This article cites 47 articles, 22 of which can be accessed free at: http://learnmem.cshlp.org/content/17/8/381.full.html\#ref-list-1

License

Email Alerting Receive free email alerts when new articles cite this article - sign up in the box at the Service top right corner of the article or click here. 